Check for updates

Cite this: RSC Adv., 2018, 8, 3646

Received 9th November 2017

Accepted 26th December 2017

DOI: $10.1039 / c 7 r a 12280 b$

rsc.li/rsc-advances

\section{Synthesis and antimicrobial effects of highly dispersed, cellulose-stabilized silver/cellulose nanocomposites}

\author{
N. S. Alahmadi, ${ }^{a}$ J. W. Betts, ${ }^{\text {b }}$ T. Heinze, ${ }^{\text {C }}$ S. M. Kelly, (D) ${ }^{* a}$ A. Koschella ${ }^{c}$ \\ and J. D. Wadhawan (iD d
}

Small, spherical silver nanoclusters were synthesised on the surface of paper as a model cellulosic fibre substrate by a standard chemical reduction method. The concentration of the silver nanoclusters on the substrate surface is roughly proportional to the initial silver salt concentration. However, there is a noticeable degree of nanocluster aggregation to larger agglomerates. The addition of small amounts of $\alpha$-cellulose, carboxymethyl cellulose or aminocellulose during the synthesis of the silver/cellulose nanocomposites suppresses this aggregation and significantly increases the concentration of the silver nanoclusters on the surface of the fibres of cellulose. These small, surface-stabilised silver nanoclusters, with the desired size and morphology, deposited from aqueous solutions on the surface of cellulosic cotton fibres, show enhanced antibacterial activity against MRSA compared to that of the corresponding silver/cotton nanocomposites prepared in the absence of a cellulosic surface stabiliser.

\section{Background}

Silver is one of the most intensively studied noble metals due to a combination of physical property values of real relevance to a wide range of major industrial and medical applications. ${ }^{\mathbf{1 - 9}}$ Silver nanoclusters, such as spherical nanoparticles, nanowires, nanobelts, nanocubes and nanoplates with different sizes and shapes, have been synthesised using a wide variety of synthetic methods and approaches. ${ }^{\mathbf{1 0}-23}$ Some of the most common applications of metallic silver and silver ions are as anti-septic and anti-microbial agents., ${ }^{\mathbf{1 , 2 4 - 2 9}}$ There has been intense interest in using silver compounds as broad-spectrum antimicrobial agents especially due to the increasing prevalence of anti-microbial resistant infections, such as MRSA, in medical environments, $c f$., hospitals, and in the treatment of severe burns. The antimicrobial action of silver compounds may well be linked to the interaction of silver ions with bacterial respiratory enzymes and the binding of silver with the bacterial cell wall inhibiting the uptake of nutrients. ${ }^{30}$

Silver nanoclusters exhibit improved antimicrobial action compared to that of conventional silver compounds due to their

${ }^{a}$ School of Physical Sciences and Mathematics, Chemistry, University of Hull, Cottingham Road, Hull, HU6 7RX, UK. E-mail: s.m.kelly@hull.ac.uk

${ }^{b}$ School of Veterinary Medicine, University of Surrey, Daphne Jackson Road, Guildford, GU2 $7 A L, U K$

'Institute for Organic Chemistry and Macromolecular Chemistry, Center of Excellence for Polysaccharide Research, Friedrich Schiller University of Jena, Humboldtstraße 10, D-07743 Jena, Germany

${ }^{d}$ School of Computer Science and Engineering, Chemical Engineering, University of Hull, Cottingham Road, Hull, HU6 $7 R X, U K$ greater overall surface-area-to-volume ratio and, thus, high degree of bioavailability. ${ }^{31}$ Silver nanoclusters of various size and shapes penetrate bacterial cell membranes and interfere with the respiratory chain- and cell-division eventually leading to cell death. ${ }^{32-34}$ Electronic interactions between bacteria and nanoparticles with a small diameter $(d \leq 10 \mathrm{~nm})$ may result in a greater degree of antibacterial action. ${ }^{35}$ Although silver compounds can be used as the active ingredient at relatively low concentrations in disinfectants against microorganisms, they can also exhibit toxic activity in human cells, resulting in argyrosis, at higher concentrations in preparations designed for medical applications. ${ }^{36}$ Fortuitously, silver nanoclusters have not been reported to be particularly toxic. Furthermore, relatively low concentrations can be used due to the nanoparticles high degree of bioavailability and antimicrobial action, thereby reducing the risk of any significant side effects resulting from silver-induced cytotoxicity.

Agglomeration (aggregation) is an important issue associated with the preparation and application of nanoclusters, since it is energetically favourable for nanoparticles to aggregate and/or fuse together in order to reduce the very large and active surface area. ${ }^{37}$ In order to inhibit aggregation, dispersions of nanoparticles and nanoclusters are often stabilised using a wide variety of surface-active additives as a capping agent. ${ }^{38,39}$ This approach also facilitates the self-organisation of the surfacestabilised nanoparticles and nanoclusters and also provides a means of control of their morphology, in terms of size, shape as well as the nature and degree of crystallinity, by influencing their kinetic growth. ${ }^{\mathbf{4 0 - 4 2}}$ Examples of silver nanoclusters stabilised by cellulosic derivatives include silver/carboxymethyl 
cellulose (CMC) nanocomposites, silver/porous cellulose fibres, nanoporous silver/cellulose gels, silver/microbial cellulose filters, silver/microcrystalline cellulose nanocomposites, silver/ bacterial cellulose nanocomposites, silver/oxidized cellulose microfibrils and silver/aminocellulose nanocomposites..$^{4-48}$ Unfortunately, the cellulose derivatives used in the synthesis of these silver/cellulose nanocomposites remain in the solid or swollen state as a consequence of their insolubility in water and also in most common organic solvents. ${ }^{49}$

In this paper we study the use of small amounts of $\alpha$-cellulose, carboxymethyl cellulose and aminocellulose as low-cost, water-soluble additives in the simple, environmentally friendly synthesis of silver/cellulose and silver/cotton nanocomposites. The purpose of the presence of these additives is to suppress nanocluster aggregation and thereby to stabilise a thin, uniform coating of surface-stabilised silver nanoclusters at high concentration on the surface of cellulosic fibres with the optimal diameter $(d \leq 10 \mathrm{~nm})$ for effective antimicrobial action, especially against multidrug resistant Staphylococcus aureus (MRSA), in a simple and cost-effective manner. Paper is used as a simple, planar model substrate, composed of cellulosic fibres, to enable and facilitate characterisation of the nature and concentration of the silver nanoclusters on the surface of the fibres. Cellulosic cotton fibres, used in wound dressings for example, infused with these surface-stabilised silver nanoclusters are then tested for antibacterial activity against MRSA.

\section{Experimental}

\section{Chemicals and materials}

Carboxymethyl cellulose (CMC) and aminocellulose (AMC, 1\%, $\mathrm{DS}=0.5$ ) were provided by the University of Jena and TITK, Germany. Silver nitrate $\left(\mathrm{AgNO}_{3}\right.$, purity $\left.>99 \%\right)$, sodium borohydride $\left(\mathrm{NaBH}_{4}\right.$, purity $\left.>96 \%\right)$, ethanol and powdered $\alpha$-cellulose were purchased from Lancaster, Fluka, Fisher Scientific and Sigma Aldrich, respectively. Metal salts and other regents were used without further purification. Deionised water was used for all experiments. The antimicrobial activity of silver nanoparticles was assessed against meticillin-resistant Staphylococcus aureus (MRSA) (NCTC 12493) purchased from the Health protection agency laboratories, Porton Down, UK. MRSA was selected due to its often-high presence in hospital and community settings and its association with antibiotic resistance and potentially lifethreatening infections. All agar and media including IsoSensitest agar and broth were purchase from Oxoid, UK.

\section{In situ synthesis of silver nanoclusters}

Cellulose paper was immersed in aqueous silver nitrate solution $(10 \mathrm{~mL})$ of different concentrations $(10 \mathrm{mM}, 44.5 \mathrm{mM}$ and 100 $\mathrm{mM}$ ) at room temperature. Contact was maintained for $3 \mathrm{~min}$ before removing the paper sample and washing it with ethanol for about $30 \mathrm{~s}$. It was then placed in sodium borohydride solution $(10 \mathrm{~mL})$ for $10 \mathrm{~min}$, removed and rinsed with deionised water for $1 \mathrm{~min}$ then dried overnight under vacuum at room temperature. Fig. 1(a) displays a schematic representation of this simple process.

\section{Synthesis of cellulose-stabilised silver/cellulose nanocomposites}

Silver nitrate $(100 \mathrm{mM})$ and a fixed amount $(1 \mathrm{mg})$ of powdered $\alpha$-cellulose, carboxymethyl cellulose (CMC) or aminocellulose (AMC) were dissolved in of deionised water $(10 \mathrm{~mL})$. A standard

Table 1 Silver/cellulose nanocomposites 1-3, prepared at different concentrations of silver nitrate $(10 \mathrm{mM}, 44.5 \mathrm{mM}$ and $100 \mathrm{mM}$, respectively), and cellulose-, CMC- and AMC-stabilised silver/cellulose nanocomposites 4-6

\begin{tabular}{llll}
\hline Sample & $\mathrm{AgNO}_{3}(\mathrm{mM})$ & Stabiliser $(1 \mathrm{mg})$ & $\begin{array}{l}\mathrm{Ag}(\% \\
\mathrm{wt} / \mathrm{wt})\end{array}$ \\
\hline $\mathbf{1}$ & 10 & - & 0.31 \\
$\mathbf{2}$ & 44.5 & - & 0.89 \\
$\mathbf{3}$ & 100 & - & 1.6 \\
$\mathbf{4}$ & 100 & Cellulose & 2.33 \\
$\mathbf{5}$ & 100 & CMC & 3.58 \\
$\mathbf{6}$ & 100 & AMC & 1.07
\end{tabular}

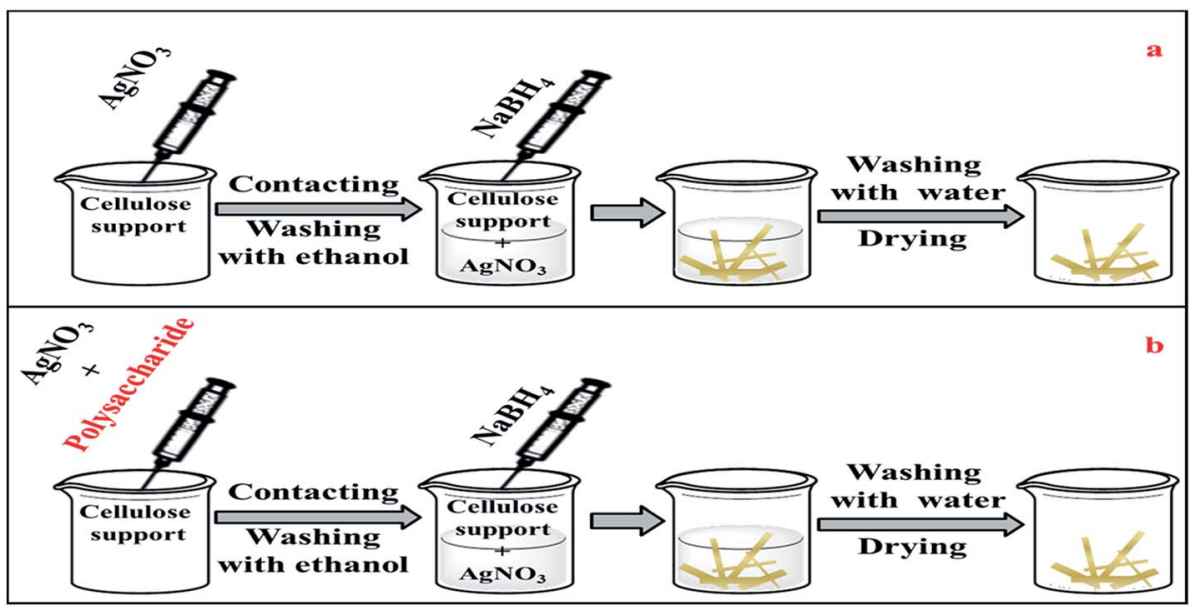

Fig. 1 A schematic representation of the synthesis process (a) without polysaccharide, (b) in the presence of polysaccharide. 


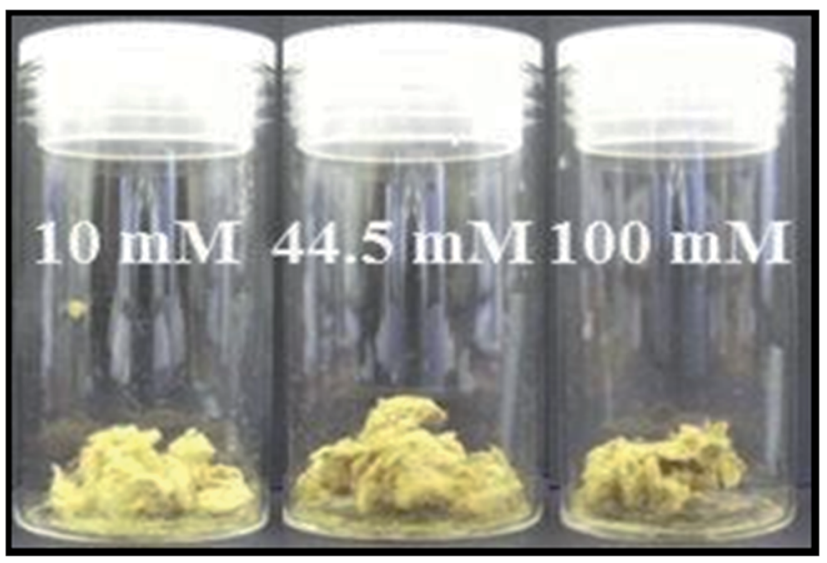

Fig. 2 Images of the silver/cellulose nanocomposites 1-3, prepared at different concentrations of silver nitrate $(10 \mathrm{mM}, 44.5 \mathrm{mM}$ and $100 \mathrm{mM}$, respectively).

cellulose paper sample was then immersed in this solution for ca. 3 minutes, then removed, rinsed with ethanol and then placed in a $(100 \mathrm{mM})$ sodium borohydride solution $(10 \mathrm{~mL})$ for a further $10 \mathrm{~min}$. The paper sample was removed and rinsed with deionised water for $1 \mathrm{~min}$ and then dried overnight under vacuum at room temperature. Fig. 1(b) represents a schematic representation of this process.

\section{Synthesis of cellulose-stabilised silver/cotton nanocomposites}

Silver nitrate $(100 \mathrm{mM})$ and a fixed amount $(1 \mathrm{mg})$ of powdered cellulose, carboxymethyl cellulose (CMC) or aminocellulose (AMC) were dissolved in deionised water $(10 \mathrm{~mL})$. The solution was stirred for $3 \mathrm{~min}$ and then sodium borohydride solution (10 $\mathrm{mL}$ ) was added to the solution. A cotton fibre was immersed in the aqueous dispersions of surface-stabilised silver nanoclusters for 24 hours. The fibre was then rinsed with deionised water for $1 \mathrm{~min}$ and dried overnight under vacuum at room temperature.

NB: the analogous silver/cellulose and silver/cotton nanocomposites were prepared in similar ways in the absence of cellulose derivatives as reference materials.

\section{Antibacterial assays}

The diffusion assay was chosen to determine the antibacterial activities of cotton fibres infused with surface-stabilised silver nanoclusters, with $\alpha$-cellulose, carboxymethyl cellulose or aminocellulose as the surface stabiliser, as well as that of the reference non-surface-stabilised silver/cotton nanocomposite. Individual plates of Iso-Sensitest agar were inoculated with MRSA, using the standardized method by Moosdeen et al. ${ }^{50}$ Samples of cotton fibres infused with the four types of silver nanoclusters were added to each plate. Agar plates were then incubated at $37^{\circ} \mathrm{C}$ for $24 \mathrm{~h}$. Three replicates were performed for each agar plate produced in order to confirm any apparent results. The method of assessing antibacterial activity was based on that used by Pollini et al. ${ }^{51}$ where good action was the presence of an inhibition zone $>1 \mathrm{~mm}$, fairly good action was the presence of an inhibition zone $<1 \mathrm{~mm}$, sufficient action where the absence of growth on the sample was observed, limited action described limited bacterial growth on the sample and not sufficient described no antibacterial action where the sample was either partially or totally overgrown by bacteria.

Broth microdilution assays were performed in order to determine the minimum bactericidal and inhibitory concentrations (MBC/MIC), of each silver nanocomposite in a liquid culture against MRSA NCTC 12493. For each stabilized silver nanoparticle sample $200 \mu \mathrm{L}$ was pipetted into wells on row 1 of a 96 well cell culture plate before doubling dilutions were performed along the plate, by pipetting $100 \mu \mathrm{L}$ of the nanoparticle solutions into row 2 containing $100 \mu \mathrm{L}$ of Iso-Sensitest broth.

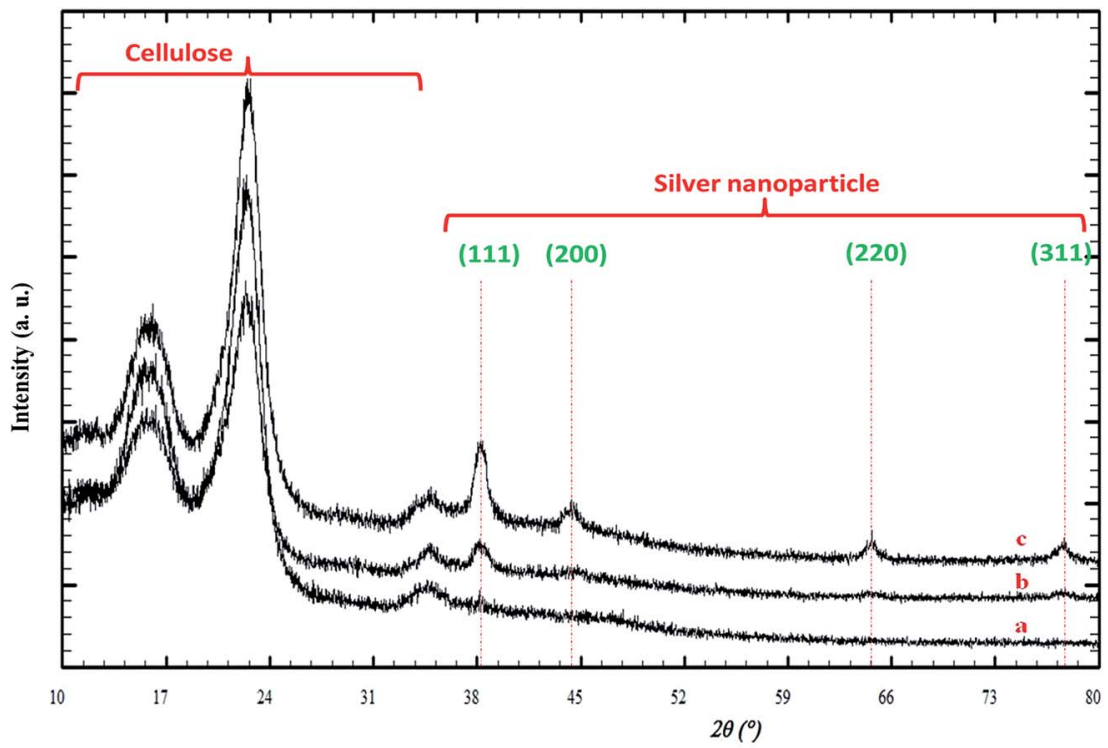

Fig. 3 PXRD patterns of the silver/cellulose nanocomposites 1-3 prepared using different concentrations of silver nitrate solution (a) 10 mM, (b) $44.5 \mathrm{mM}$ and (c) $100 \mathrm{mM}$, respectively. 
This was repeated from along rows 3 to 11 . No nanoparticles were added to wells in row 12, to provide a positive growth control. MRSA inoculant was pipetted into each well as a $100 \mu \mathrm{L}$ volume of a 0.5 MacFarland suspension in Iso-Sensitest broth. All microtitre plates were incubated in a rocking incubator at $37{ }^{\circ} \mathrm{C}$ for 24 hours. Following the incubation all wells were observed for turbidity. The MIC was recorded, as the well with the lowest concentration of silver nanocomposites were no turbidity (visible growth) was observed. The bactericidal concentration was determined by removing a fixed volume $(2 \mu \mathrm{L})$ of the broth from each microtitre plate well. Each of these samples was spotted onto Iso-Sensitive agar and then all of the agar plates were then incubated for a further for $18 \mathrm{~h}$ at $37^{\circ} \mathrm{C}$. The wells with the lowest silver nanocomposite concentration, where no growth was observed, was recorded as the MBC. Three repeat experiments were conducted for each sample solution of silver nanoparticles against MRSA in order to confirm each result.

\section{Characterisations of silver/cellulose nanocomposites}

The silver cellulose nanocomposites were characterised by inductively coupled plasma mass spectrometry (ICP-MS), performed on a Perkin Elmer DRC ICPMS ultra trace metal analyser. The structure of the silver/cellulose nanocomposites was studied using X-ray powder diffraction using a Siemens D 5000 X-ray diffractometer fitted with Ni-filtered $\mathrm{Cu} \mathrm{K} \alpha(\lambda=0.154 \mathrm{~nm})$ at room temperature. The diameter $d$ of the spherical nanocrystallites was estimated by using Scherrer's formula, $d=k \lambda$ / $B \cos \theta$, where $\lambda=$ wavelength of X-rays $(0.154 \mathrm{~nm}), K$ a constant (0.9), $B$ is the full-width half-maximum of intensity (FWHM) and $\theta$ is the peak angle (measured in radians). The average of all four silver peaks observed was used to determine the Ag nanocluster diameter, $d$. Ultraviolet-visible reflectance spectra were recorded using a Varian Cary 5E Ultraviolet-visible-NIR spectrometer, equipped with a Harrick Praying Mantis solid-state diffuse reflectance accessory. Transmission Electron Microscopy (TEM) images were obtained using a JEOL 2010 transmission electron microscope (JEOL UK Ltd. Welwyn Garden City) running at $120 \mathrm{kV}$.

\section{Results and discussion}

\section{Silver/cellulose nanocomposites}

No visible change, i.e., in colour, was observed when the silver nanoclusters were immobilised on the surface of the standard paper sample. However, each of the treated paper samples rapidly turned black on the addition of the sodium borohydride solution, which is indicative of the reduction of silver ions to form small silver nuclei in the silver/cellulose nanocomposites 1-3, prepared at different concentrations of silver nitrate (10 $\mathrm{mM}, 44.5 \mathrm{mM}$ and $100 \mathrm{mM}$, respectively). Over time these paper samples became lighter in colour, which may well be

Table 2 The diameter, $d$, of the silver/cellulose nanocomposites 1-3, prepared at different concentrations of silver nitrate $(10 \mathrm{mM}, 44.5 \mathrm{mM}$ and $100 \mathrm{mM}$, respectively), and that of $\alpha$-cellulose-, CMC- and AMCstabilised silver/cellulose nanocomposites 4-6

\begin{tabular}{llll}
\hline Sample & $\mathrm{AgNO}_{3}(\mathrm{mM})$ & Stabiliser $(1 \mathrm{mg})$ & Diameter $(\mathrm{nm})$ \\
\hline $\mathbf{1}$ & 10 & - & 10 \\
$\mathbf{2}$ & 44.5 & - & 11 \\
$\mathbf{3}$ & 100 & - & 10 \\
$\mathbf{4}$ & 100 & Cellulose & 9 \\
$\mathbf{5}$ & 100 & CMC & 12 \\
$\mathbf{6}$ & 100 & AMC & 12
\end{tabular}

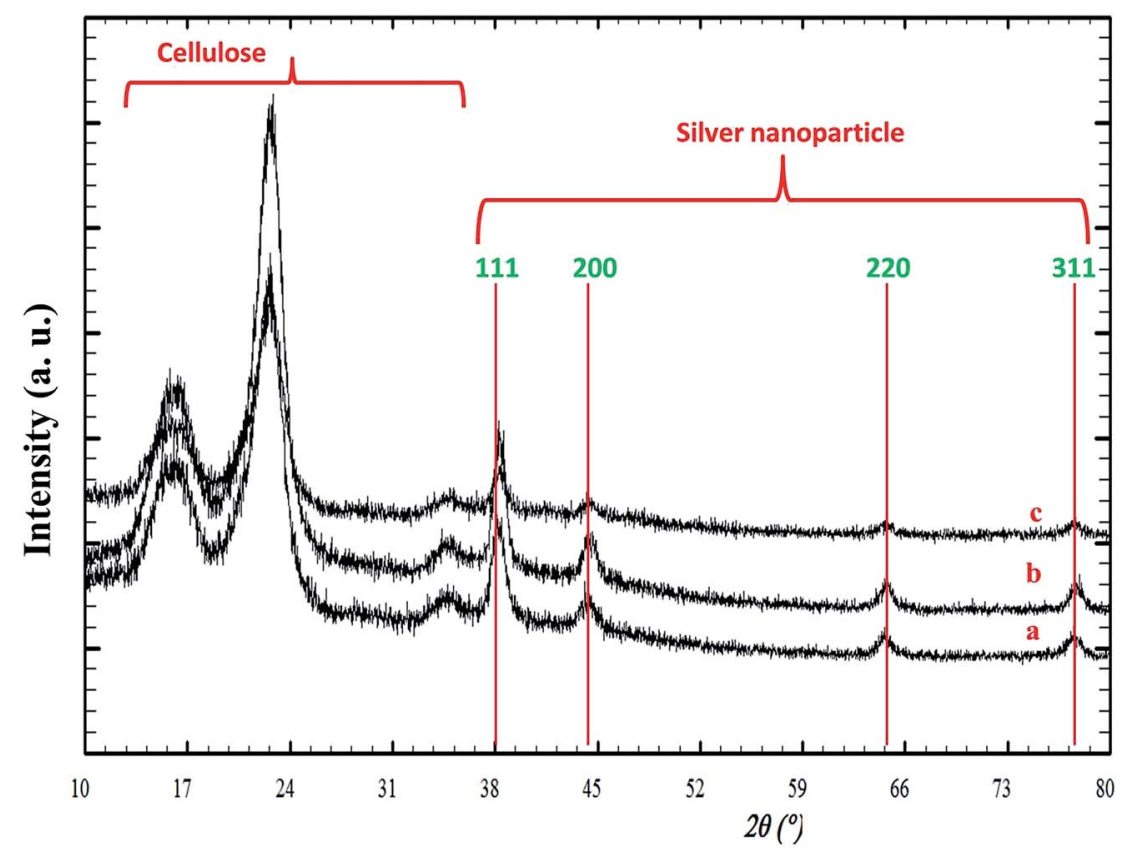

Fig. 4 PXRD patterns of silver/cellulose nanocomposites 4-6 synthesised using a standard silver nitrate solution (100 mM) with different cellulosic derivatives as an additional stabiliser, i.e., (a) $\alpha$-cellulose (b) CMC and (c) AMC, respectively. 
indicative of growth in the diameter of the nanoparticles. A homogeneous yellow colour, attributable to surface plasmon resonance (SPR) of the silver nanoclusters, was observed over the treated surface of the silver/cellulose nanocomposites 1-3 after drying the samples overnight under vacuum, which also suggests that the silver nanoparticles are well dispersed over the surface of these simple cellulose substrates. An increase of the concentration of the initial silver nitrate solution $(10 \mathrm{mM}$, $44.5 \mathrm{mM}$ and $100 \mathrm{mM}$ ), see Table 1 , to produce the silver/ cellulose nanocomposites 1-3, respectively, results in a change of colour of the product from light to dark yellow, see Fig. 2.

\section{Surface-stabilised silver/cellulose nanocomposites}

No colour change was observed on the addition of the $\alpha$-cellulose, carboxymethyl cellulose, or aminocellulose and the silver nitrate solution to the standard paper sample. However, each of the paper samples turned a dark shade of yellow on reduction of the silver nitrate solution using the sodium borohydride solution $(100 \mathrm{mM})$ to produce the $\alpha$-cellulose -, CMC- and AMCstabilised silver/cellulose nanocomposites 4-6, respectively, see Table 1.

\section{Characterisations of silver/cellulose nanocomposites}

\section{Inductively coupled plasma (ICP)}

Table 1 records the amount of silver nanoparticles adsorbed on the surface of the silver/cellulose nanocomposites 1-3, prepared at different concentrations of silver nitrate $(10 \mathrm{mM}, 44.5 \mathrm{mM}$ and $100 \mathrm{mM}$, respectively), and cellulose-, CMC- and AMC-

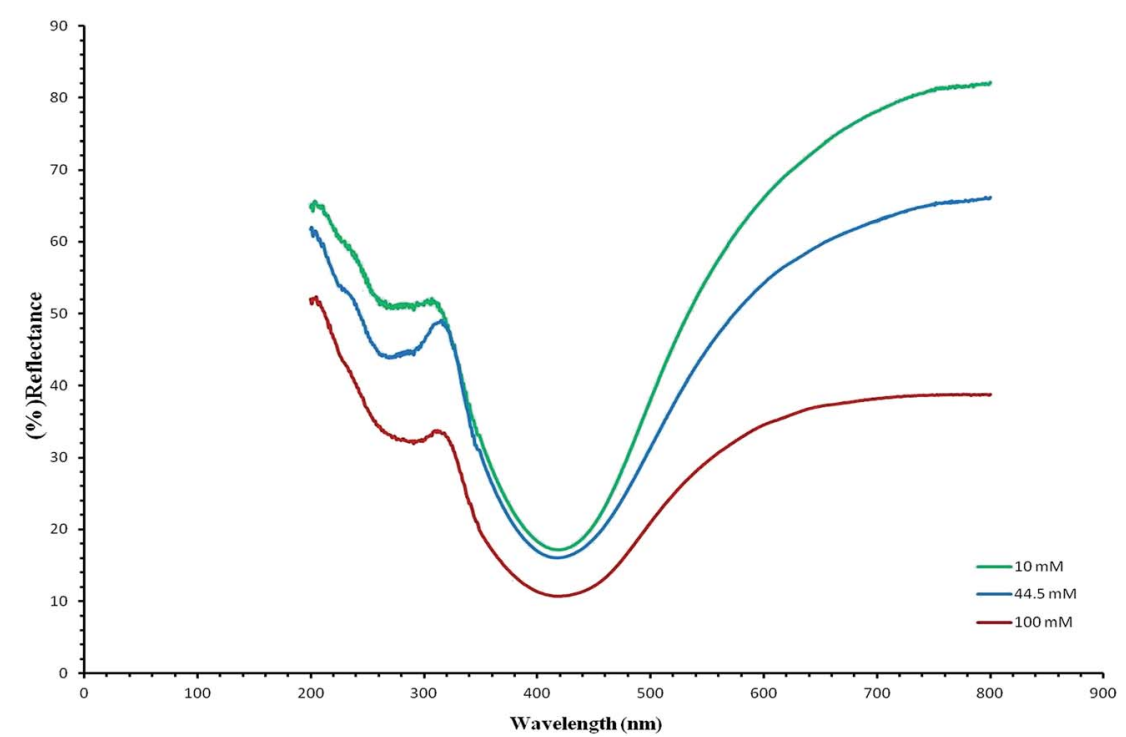

Fig. 5 UV/vis reflectance spectra (green, blue and red) of the silver/cellulose nanocomposites 1-3, prepared at different concentrations of silver nitrate solution (10 mM, $44.5 \mathrm{mM}$ and $100 \mathrm{mM}$, respectively).

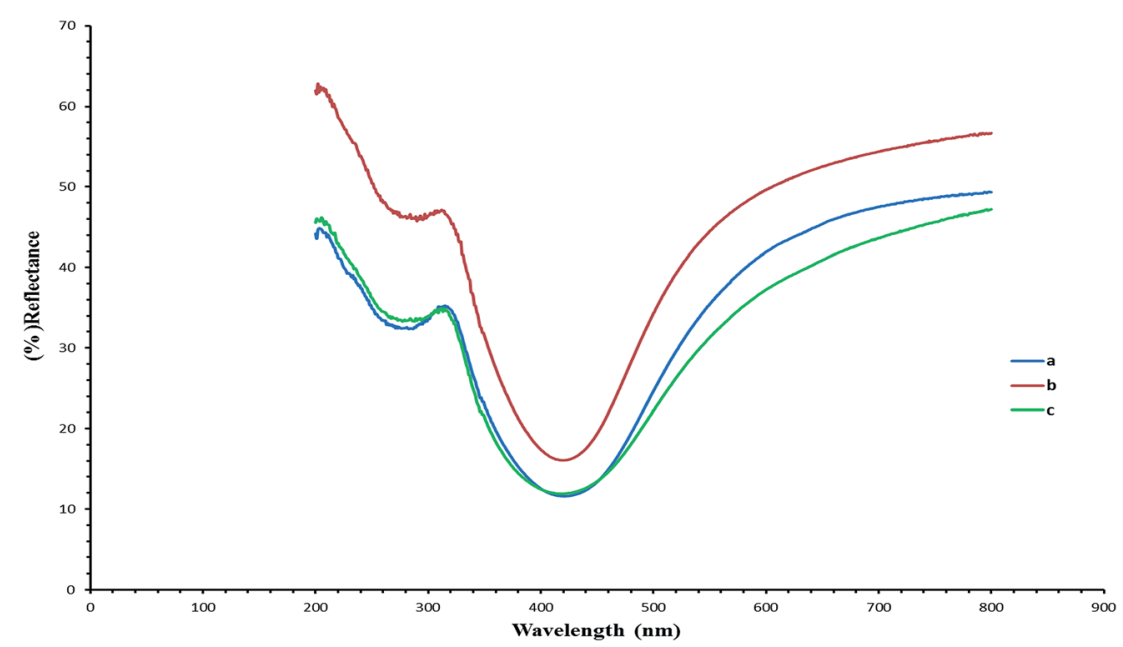

Fig. $6 \mathrm{UV} / \mathrm{vis}$ reflectance spectra (blue, red and green) of the surface-stabilised silver/cellulose nanocomposites 4-6 synthesised using a standard silver nitrate solution $(100 \mathrm{mM})$ with a fixed amount $(1 \mathrm{mg})$ of a cellulose or cellulose-derivative as an additional surface-stabiliser, i.e., (a) powdered $\alpha$-cellulose (b) CMC and (c) AMC, respectively. 
stabilised silver/cellulose nanocomposites 4-6, determined using ICP analysis. The quantity of silver nanoclusters present on the paper sample appears to be directly proportional to the concentration of silver nitrate in the precursor solution. The concentration (wt\%/wt\%) of silver nanoclusters is higher in the silver/cellulose nanocomposites 4-6 prepared in the presence of $\alpha$-cellulose, CMC and AMC, see Table 1, than that determined for the silver/cellulose nanocomposites 3, prepared at the same concentration of silver nitrate $(100 \mathrm{mM})$, in the absence of any other cellulose-related additives.

\section{Powder X-ray diffraction}

Fig. 3 illustrates the XRD patterns for the silver/cellulose nanocomposites 1-3 prepared using different concentrations of silver nitrate solution (a) $10 \mathrm{mM}$, (b) $44.5 \mathrm{mM}$ and (c) $100 \mathrm{mM}$. The first three peaks are attributed to cellulose and four peaks at Bragg angles $38^{\circ}, 44^{\circ}, 64^{\circ}$ and $77^{\circ}$ are attributed to the crystallographic planes of face-centred cubic (fcc) silver crystals. These peaks agree with the peaks of pure silver metal [JCPDS card no. 01-1167]. No other peaks due to the presence of other elements are observed. This combination of peaks indicates that the nanoclusters consist of pure metallic silver with a high degree of crystallinity. It is fairly typical for the peaks at shorter wavelength ( 2 theta) to be more intense and these typically correspond to the lower Miller indices. The presence of the 111 peak suggests that there are silver atoms lying on the plane defined by those Miller indices. The XRD pattern shown in Fig. 3 is typical of both metallic silver and silver nanoparticles. ${ }^{52}$ The diameter of the silver nanoclusters on the surface of the silver/cellulose nanocomposites 1-3, calculated using Scherrer's equation, is similar in each case $(d=10-11 \mathrm{~nm})$.

Fig. 4(a-c) displays the PXRD patterns of the silver/cellulose nanocomposites 4-6 synthesised with different cellulose
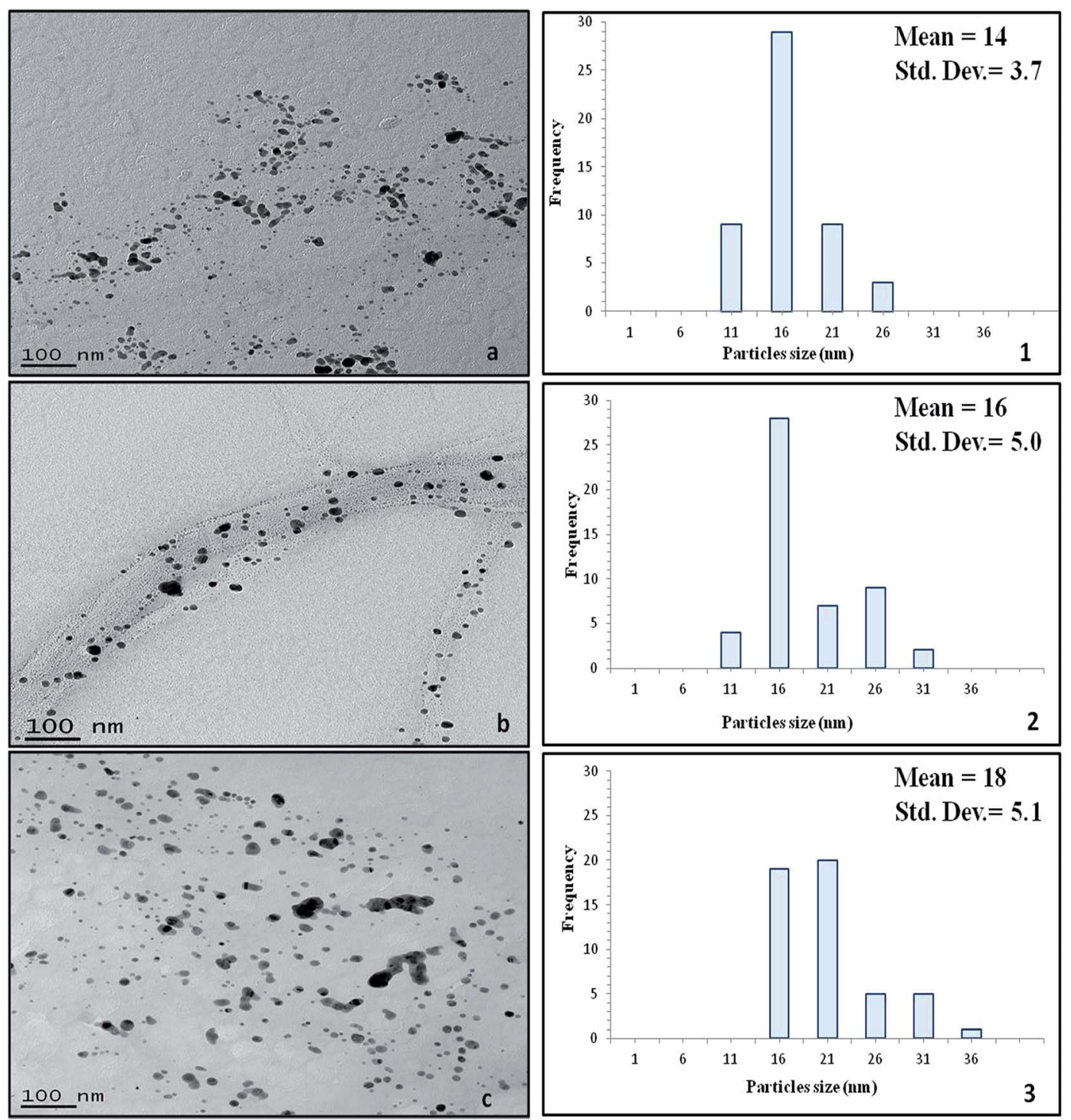

Fig. 7 TEM images of the silver/cellulose nanocomposites 1 (panel a), 2 (panel b) and 3 (panel c) prepared using three different concentrations of silver nitrate solution (10 mM, $44.5 \mathrm{mM}$ and $100 \mathrm{mM}$, respectively). 
derivatives as an additional stabiliser, i.e., (a) powdered cellulose (b) CMC and (c) AMC, respectively, using a standard silver nitrate solution (100 $\mathrm{mM})$. There is no shift in peak position for either silver or cellulose, which implies no chemical changes in either. The observed differences in relative intensity of the silver peaks in Fig. 4(a-c) are attributable to higher concentrations of surface-stabilised silver nanoclusters in the silver/cellulose nanocomposites 4-6, prepared with the three different cellulose derivatives as an additional stabiliser, i.e., (a) powdered cellulose (b) CMC and (c) AMC, respectively, which is consistent with the ICP results shown in Table 1 . The diameter $(d=9-12$ $\mathrm{nm}$ ) of the silver nanoclusters of the silver/cellulose nanocomposites 4-6 is very similar to that $(d=10 \mathrm{~nm})$ of the silver/ cellulose nanocomposites 3 prepared using the same silver nitrate solution $(100 \mathrm{mM})$ see Table 2 .

\section{Ultraviolet-visible spectroscopy}

Fig. $5 \mathrm{UV} /$ vis shows the reflectance spectra (green, blue and red) of the silver/cellulose nanocomposites $\mathbf{1 - 3}$, prepared at different concentrations of silver nitrate solution $(10 \mathrm{mM}$, $44.5 \mathrm{mM}$ and $100 \mathrm{mM}$, respectively). These peaks are attributable to Surface Plasmon Resonance effects due to the quantumconfined nature of the silver nanoclusters stabilised on the paper substrate surface and the positions of the adsorption peaks depend on the size and shape of the nanoparticle.,19 The reflectance maxima $\left(\lambda_{\max }\right)$, shown in the spectra, are consistent with a limited degree of dispersity in the size of the silver nanoclusters, see further discussion below. At the same time the intensity of peaks in the reflectance spectra (green, blue and red) of the silver/cellulose nanocomposites 1-3 is proportional to the concentration of the silver nitrate solution $(10 \mathrm{mM}$, $44.5 \mathrm{mM}$ and $100 \mathrm{mM}$, respectively).

Fig. 6 demonstrates the UV/vis reflectance spectra (blue, red and green) for the silver/cellulose nanocomposites 4-6, prepared using the standard silver nitrate solution $(100 \mathrm{mM})$ in the presence of a fixed amount $(1 \mathrm{mg})$ of powdered $\alpha$-cellulose, carboxymethyl cellulose or aminocellulose as a surfacestabiliser. The reflectance spectra and maxima $\left(\lambda_{\max }\right)$ observed for the silver/cellulose nanocomposites 4-6, are similar to those determined for the silver/cellulose nanocomposites 1-3 prepared in the absence of a cellulosic surface-stabiliser. These results are in agreement with the spherical shape, size $(d=9-12$ $\mathrm{nm}$ ) and limited dispersity of the silver nanoparticles as determined using PXRD, see Fig. 3 and 4, and the TEM results shown in Fig. 7 and 8, see below. These observations indicate that the presence of additional amounts of $\alpha$-cellulose, CMC or AMC, as a surface stabiliser, does not affect the morphology of the silver nanoparticles in a significant manner. However, the relative intensity of the peaks varies directly and proportionately with the quantity of the surface-stabilised silver nanoparticles in the silver/cellulose nanocomposites 4-6, which is consistent with the PXRD spectra, see Fig. 3 and 4 and with the ICP results, see Table 1.

\section{Transmission electron microscope}

The morphology (shape and size) of the silver nanoclusters formed on the surface of the cellulose fibres of the test paper sample was also investigated using TEM. Fig. 7 presents the
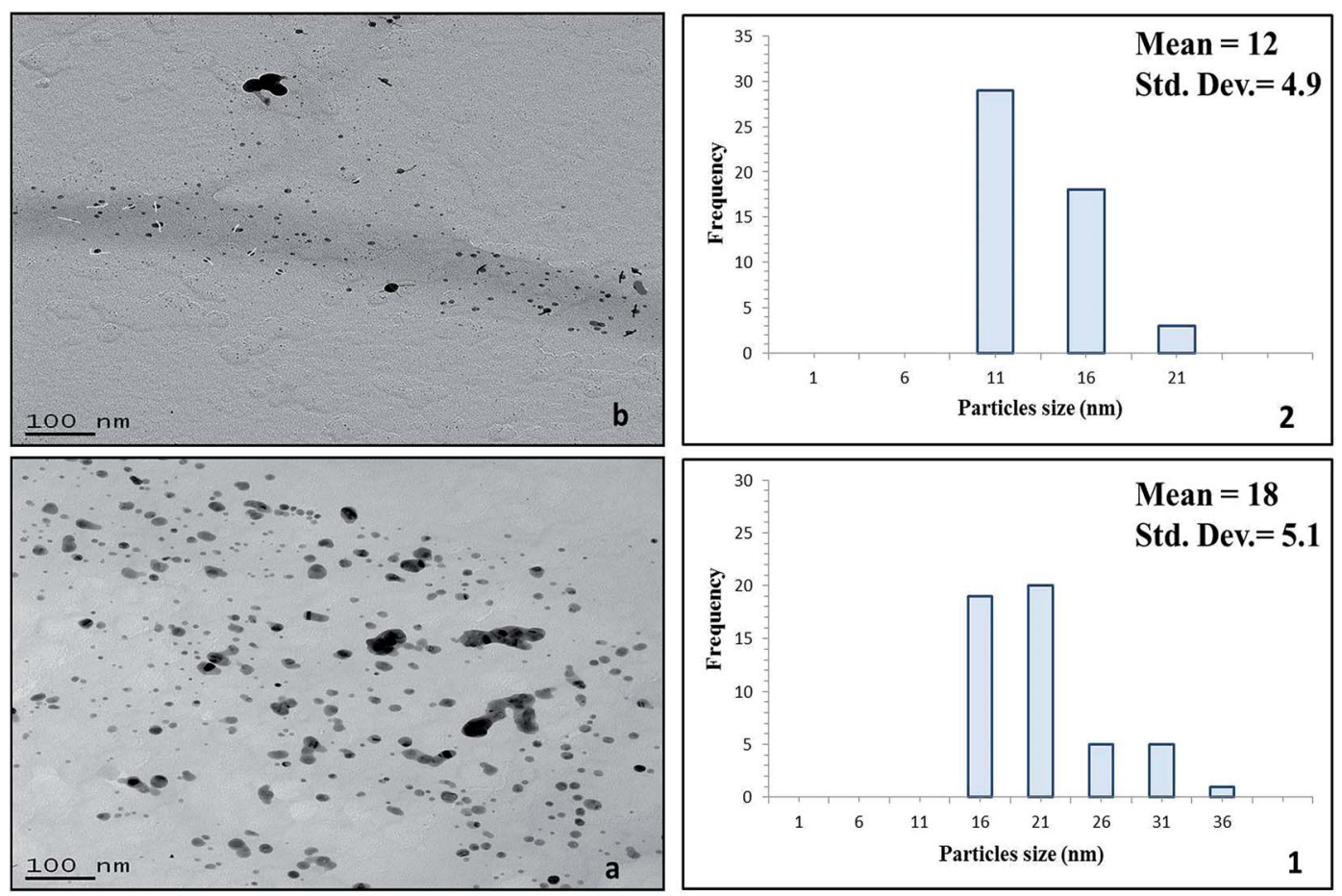

Fig. 8 TEM images of the silver/cellulose nanocomposites 3 (panel a) and 4 (panel b), prepared (8.1) in the absence of powdered $\alpha$-cellulose and (8.2) in the presence of powdered $\alpha$-cellulose, respectively. 
TEM images of the silver/cellulose nanocomposites 1-3, prepared using three different concentrations of silver nitrate solution (10 mM, 44.5 mM and $100 \mathrm{mM}$, respectively). The TEM images show that spherical silver nanoclusters are located on the surface of the cellulosic fibres of the standard paper sample used in this study to prepare the silver/cellulose nanocomposites 1-3. It appears that the morphology, i.e., the size and shape, of the small, spherical silver nanoclusters are not greatly dependent on the concentration of silver nitrate solution, although an increase in the average diameter of the silver nanocluster agglomerates $\left(d_{\text {mean }}=14,16\right.$ and $\left.18 \mathrm{~nm}\right)$ with some much larger nanoparticles $(d=31-36 \mathrm{~nm})$ is observed at higher concentrations of silver nitrate solution $(10 \mathrm{mM}, 44.5 \mathrm{mM}$ and $100 \mathrm{mM}$, respectively), see the corresponding PXRD and UV/vis spectra shown in Fig. 3 and 5, respectively. The greater values of the diameter of the silver nanocluster agglomerates observed using TEM than those ( $d=9-12 \mathrm{~nm}$ ) determined using PXRD, combined with a greater degree of polydispersity, is indicative of the aggregation of smaller silver nanoclusters into larger nano-agglomerates.

Fig. 8 show the TEM images of the silver/cellulose nanocomposites 3 and $\mathbf{4}$, prepared using the standard silver nitrate solution $(100 \mathrm{mM})$, in either the absence or the presence of powdered $\alpha$-cellulose, respectively. The silver nanoclusters are also seen to be spherical in the $\alpha$-cellulose-stabilised silver/ cellulose nanocomposites $\mathbf{4}$, but with a much lower degree of aggregation than that observed for the corresponding silver/ cellulose nanocomposite 3 , prepared in the absence of $\alpha$-cellulose in the reaction solution. The silver nanoclusters observed for the cellulose-stabilised, silver/cellulose nanocomposites 4 are much smaller on average $\left(d_{\text {mean }}=12 \mathrm{~nm}\right.$, respectively) than those $\left(d_{\text {mean }}=18 \mathrm{~nm}\right)$ determined for the silver/cellulose nanocomposites 3 , prepared in the absence of $\alpha$-cellulose, i.e., there is a much lower degree of aggregation.

\section{Scanning electron microscopy}

Fig. 9-13 presents the SEM images at two degrees of resolution (a \& b) of the silver/cellulose nanocomposites 1-3, prepared using three different concentrations of silver nitrate solution (10 $\mathrm{mM}, 44.5 \mathrm{mM}$ and $100 \mathrm{mM}$, respectively). The SEM images shown in these figures indicate that aggregates of mostly

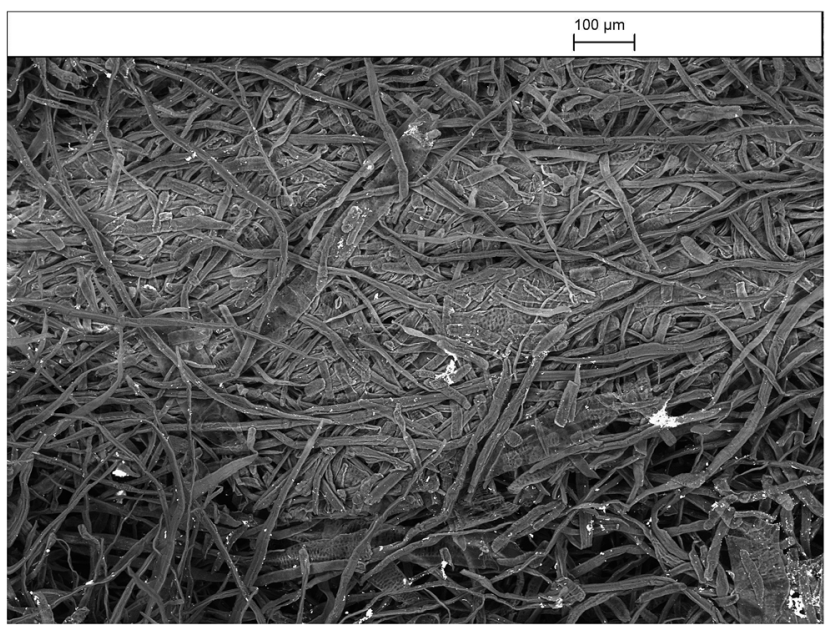

Fig. 10 SEM images of the silver/cellulose nanocomposites 2 prepared using silver nitrate solution $(45 \mathrm{mM})$.

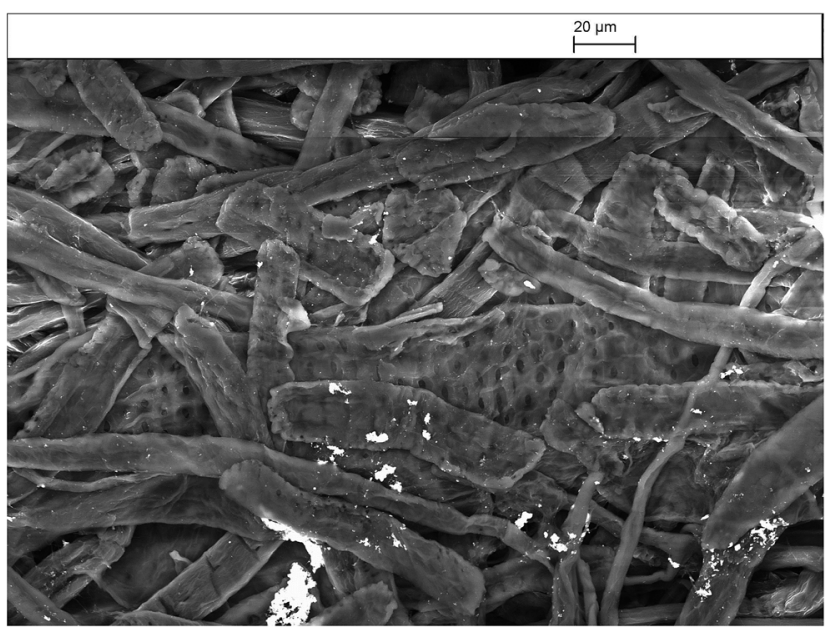

Fig. 11 SEM images of the silver/cellulose nanocomposites 2 prepared using silver nitrate solution ( $45 \mathrm{mM})$

spherical silver nanoclusters are located on the surface of the cellulosic fibres of the standard paper sample and that the concentration of the silver/cellulose nanocomposites 1-3 on the
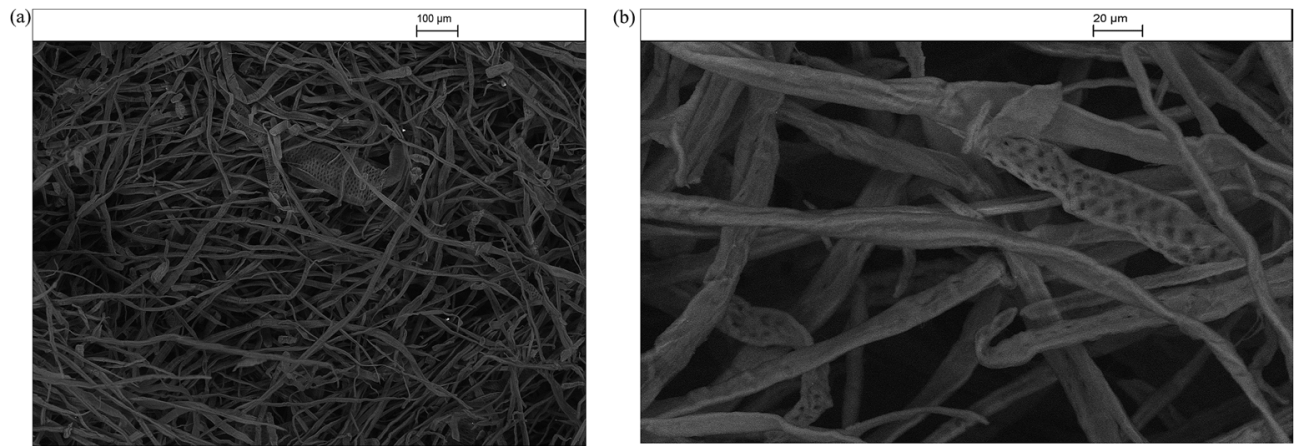

Fig. 9 (a). SEM images of the silver/cellulose nanocomposites 1 prepared using silver nitrate solution (10 mM). (b). SEM images of the silver/ cellulose nanocomposites 1 prepared using silver nitrate solution $(10 \mathrm{mM})$. 


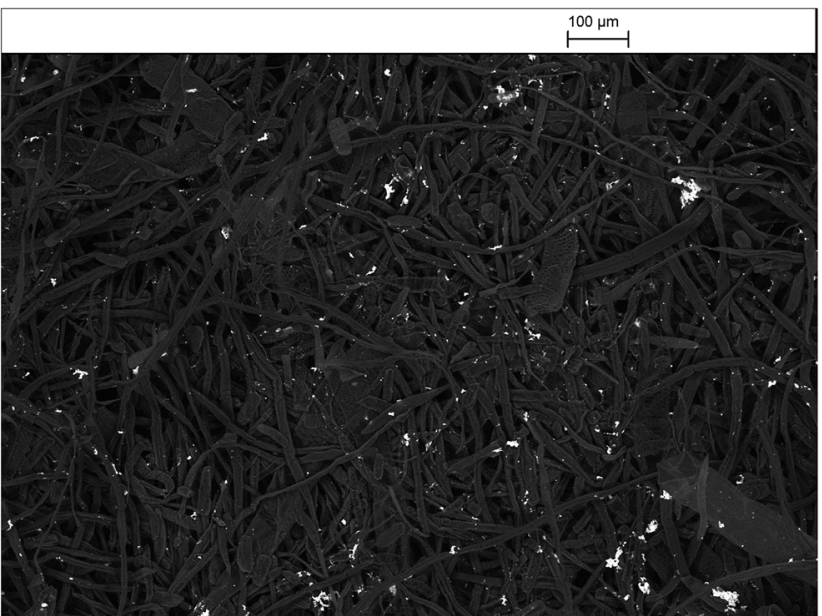

Fig. 12 SEM images of the silver/cellulose nanocomposites 3 prepared using silver nitrate solution $(100 \mathrm{mM})$.

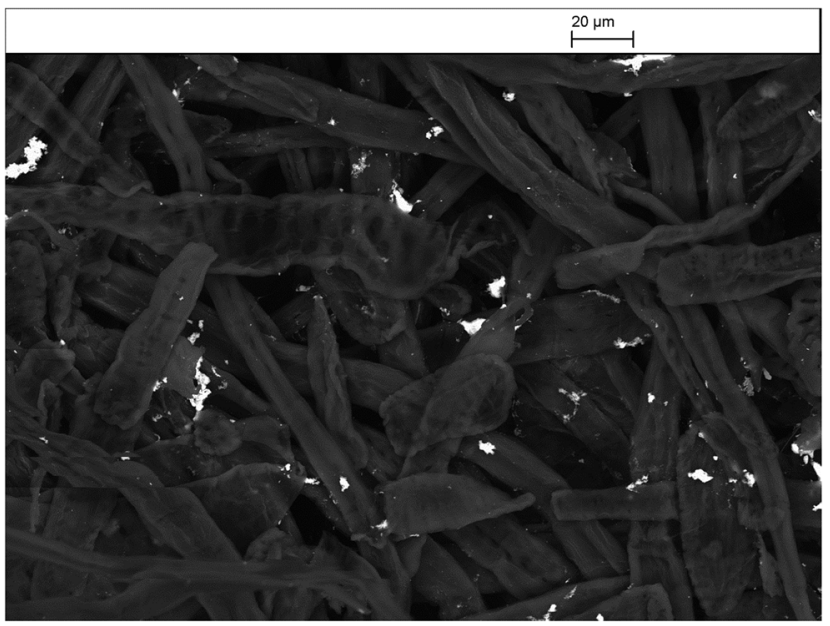

Fig. 13 SEM images of the silver/cellulose nanocomposites 3 prepared using silver nitrate solution $(100 \mathrm{mM})$.

surface of the cellulosic fibres increases with the concentration of silver nitrate solution used in the preparation of these samples.

Fig. 14 shows of the surface-stabilised silver/cellulose nanocomposite 4 synthesised using a standard silver nitrate solution $(100 \mathrm{mM})$ with a fixed amount $(1 \mathrm{mg})$ of a powdered $\alpha-$ cellulose as an additional surface-stabiliser. The silver nanoclusters are also observed to be mainly spherical in the $\alpha$ cellulose-stabilised silver/cellulose nanocomposites 4. However, a lower degree of aggregation is apparent compared to that observed for the corresponding silver/cellulose nanocomposite 3 , prepared in the absence of $\alpha$-cellulose in the reaction solution, see Fig. 12 and 13.

\section{Antibacterial assays}

The results of antibacterial testing against MRSA of the silver/ cotton nanocomposite fibres and cellulose-, CMC- and AMC-

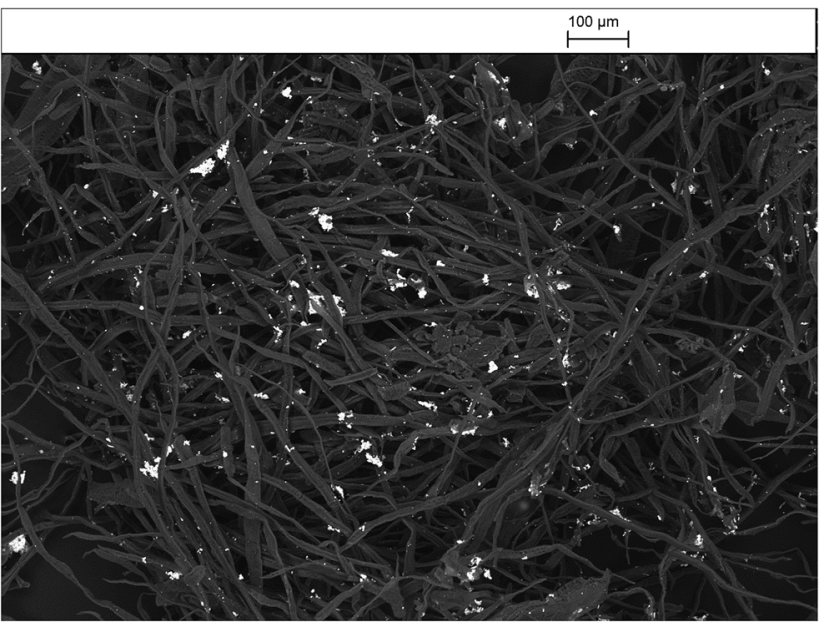

Fig. 14 SEM images of the surface-stabilised silver/cellulose nanocomposite 4 synthesised using a standard silver nitrate solution (100 $\mathrm{mM}$ ) with a fixed amount ( $1 \mathrm{mg}$ ) of a powdered $\alpha$-cellulose as a surface stabiliser.

stabilised silver/cotton nanocomposite fibres, respectively, are shown in Table 3. The antibacterial activity of the cellulose-, CMC- and AMC-stabilised silver/cotton nanocomposite fibres is superior to that of the corresponding silver/cotton nanocomposite fibres, prepared in the absence of a cellulosic derivative as stabiliser in the reaction solution. Although antibacterial action has been observed in all $\alpha$-cellulose-, CMCand AMC-stabilised silver/cotton nanocomposite fibres, see Fig. 15, the poor solubility of some of the surface-stabilised silver nanoclusters might have limited their diffusion into the agar. This lack of diffusion into the agar would also limit the zones of inhibition produced by the silver samples and thus the antibacterial action would appear lower than that in a liquid system, such as in the microtitre assays (Table 3), where the available surface area of the nanoparticles is potentially greater. MBCs and MICs for silver nanoparticles stabilised with cellulose, CMC or AMC were lower than their non-stabilised counterpart. This is likely due to stability and thus bioavailability in Iso-Sensitest broth, over the course of the assay.

Variations in antibacterial activity are likely to be due to a combination of nanocluster size and shape, concentration and the nature of the cellulosic surface stabilization, e.g., electronic interactions between bacteria and nanoparticles with a small diameter $(d \leq 10 \mathrm{~nm})$ have been related to a greater degree of antibacterial action. ${ }^{35}$ Hence, the more monodisperse

Table 3 Results of antibacterial testing of silver/cotton nanocomposites and cellulose-, CMC- and AMC-stabilised silver/cotton nanocomposites, respectively, against MRSA

\begin{tabular}{llll}
\hline Sample & $\begin{array}{l}\text { Cellulosic } \\
\text { stabiliser }\end{array}$ & $\begin{array}{l}\text { Antibacterial activity/ZOI } \\
(\mathrm{mm})\end{array}$ & MBC/MIC (mM) \\
\hline $\mathbf{3}$ & None & Sufficient/0 & $1.6 / 0.8$ \\
$\mathbf{4}$ & Cellulose & Fairly good $/ 1$ & $0.58 / 0.29$ \\
$\mathbf{5}$ & CMC & Fairly good $/ 1$ & $0.9 / 0.22$ \\
$\mathbf{6}$ & AMC & Fairly good/1 & $0.54 / 0.13$
\end{tabular}




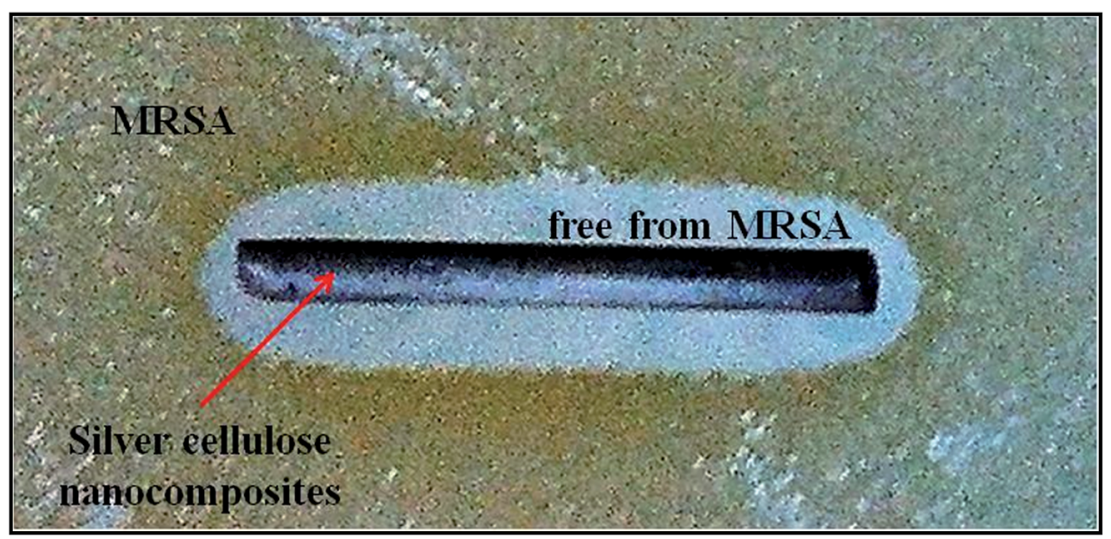

Fig. 15 The antibacterial activity of a typical surface-stabilised silver/cotton nanocomposite fibre against MRSA NCTC 12493.

the diameter of the silver nanoclusters around the optimal diameter $(d \leq 10 \mathrm{~nm})$ the greater the antibacterial action is expected to be. However, it is impossible to correlate, in any direct and meaningful fashion, the observed antibacterial activity of the silver/cellulose nanocomposites 3-6 with any individual parameter, such as size and size distribution of the silver nanoclusters. It is a complex and unpredictable function of the combination of the parameters discussed above, primarily small diameter and high concentration. Reduced bioavailability of the silver nanoclusters by either fully or partially encapsulating the silver nanoclusters could inhibit the availability of the silver ions to reach their site of action. However, if nanoparticles are stabilised on the surface of a material, any potential nanoparticle depletion would be reduced, therefore maintaining higher concentrations of surface nanoparticles, compared with non-stabilised counterparts. Stabilised nanoparticles would also remain in solution longer than those without stabilising agents such as CMC- or AMC-, maintaining the concentration of available nanoparticles, resulting in higher overall Ag+ ion release and sustained antibacterial activity over time. The cellulose derivatives themselves, present as a surface stabiliser on the silver/ nanocomposites 4-6, are expected to have a minimal effect, in addition to those discussed above, on the antibacterial activity of the silver nanocomposites as the cellulose derivatives themselves exhibit no antibacterial activity. They are also present in a very low concentration compared to that of the silver in the cellulose nanocomposites 4-6. These initial results indicate a significant potential for the use of such $\alpha$-cellulose-, CMC- and AMC-stabilised silver/cotton nanocomposite fibres in medical products, such as wound dressings and equipment, in order to aid in healing and dramatically reducing the onset of wound infection from external bacteria. Furthermore, these nanocomposite fibres could also find use as constituents of cellulosic fabrics used in medical garments in preventing the transmission of antibiotic resistant bacteria, such as MRSA, in a hospital environment.

Antibacterial activity is defined as good (Inhibition zone $>1$ $\mathrm{mm}$ ), fairly good (Inhibition zone $<1 \mathrm{~mm}$ ), sufficient (Absence of growth on the sample), limited (Limited bacterial growth on the sample) and not sufficient (Sample is either partially or totally overgrown by bacteria), $\mathrm{ZOI}=$ zone of inhibition, $\mathrm{MBC}=$ minimum bactericidal concentration, MIC $=$ minimum inhibitory concentration.

In an analogous fashion, the aqueous solutions of almost all the cellulose-stabilised silver nanoclusters, show inhibitory and bactericidal effects in the liquid culture at $50 \%$ of the concentration of cellulose-stabilized nanoclusters with potential use as antibacterial agents in medical and industrial environments.

\section{Conclusions}

Silver/cellulose nanocomposites were synthesised by a chemical reduction method using sodium borohydride as a strong reducing agent and paper as a simple model cellulosic test substrate. The quantity of silver nanoclusters deposited on the surface of the silver/cellulose nanocomposites is proportional to the initial silver salt concentration, whereas the diameter of the small, spherical nanoparticles is relativity constant. The addition of cellulosic derivatives during the synthesis of the silver nanoclusters suppresses their aggregation and significantly increases their concentration on the surface of the cellulosic fibres of the paper sample. Aqueous solutions of small, spherical, cellulose-stabilised silver nanoclusters, prepared in a similar manner, deposited on cellulosic cotton fibres, show enhanced antibacterial activity against MRSA than similar cotton fibres coated with the corresponding non-surfacestabilised silver nanoclusters.

\section{Conflicts of interest}

There are no conflicts of interest to declare.

\section{Acknowledgements}

The authors acknowledge funding from the European Union Seventh Framework Programme (FP7/2007-2013) under the Grant Agreement Number 214653 as the SURFUNCELL project. NSA would like to thank the Saudi Arabian government for financial support, Mrs A. Lowry for TEM, Mr R Knight for ICP and Dr NA Young for UV/vis spectroscopy. Dr M. Kelly is thanked for comments on the interpretation of the XRD spectra. 


\section{References}

1 J. S. Kim, E. Kuk, K. N. Yu, J.-H. Kim, S. J. Park, H. J. Lee, S. H. Kim, Y. K. Park, Y. H. Park, C.-Y. Hwang, Y.-K. Kim, Y.-S. Lee, D. H. Jeong and M.-H. Cho, Nanomedicine, 2007, 3, 95-101.

2 P. C. Lee and D. Meisel, J. Phys. Chem., 1982, 86, 3391-3395.

3 A. Panáček, L. Kvítek, R. Prucek, M. Kolář, R. Večeřová, N. Pizúrová, V. K. Sharma, T. j. Nevěčná and R. Zbořil, $J$. Phys. Chem. B, 2006, 110, 16248-16253.

4 A. I. Lukman, B. Gong, C. E. Marjo, U. Roessner and A. T. Harris, J. Colloid Interface Sci., 2011, 353, 433-444.

5 T. Maneerung, S. Tokura and R. Rujiravanit, Carbohydr. Polym., 2008, 72, 43-51.

6 N. R. Jana, L. Gearheart and C. J. Murphy, Chem. Commun., 2001, 617-618.

7 J. J. Mock, M. Barbic, D. R. Smith, D. A. Schultz and S. Schultz, J. Chem. Phys., 2002, 116, 6755-6759.

8 M. Maillard, S. Giorgio and M.-P. Pileni, J. Phys. Chem. B, 2003, 107, 2466-2470.

9 G. McNay, D. Eustace, W. E. Smith, K. Faulds and D. Graham, Appl. Spectrosc., 2011, 65, 825-837.

10 S. Schneider, P. Halbig, H. Grau and U. Nickel, Photochem. Photobiol., 1994, 60, 605-610.

11 N. Shirtcliffe, U. Nickel and S. Schneider, J. Colloid Interface Sci., 1999, 211, 122-129.

12 S. Chen and D. L. Carroll, Nano Lett., 2002, 2, 1003-1007.

13 Y. Sun, B. Mayers and Y. Xia, Nano Lett., 2003, 3, 675-679.

14 Y. Sun and Y. Xia, Adv. Mater., 2003, 15, 695-699.

15 I. Sondi, D. V. Goia and E. Matijević, J. Colloid Interface Sci., 2003, 260, 75-81.

16 B. Wiley, Y. G. Sun, B. Mayers and Y. N. Xia, Chem.-Eur. J., 2005, 11, 454-463.

17 I. Washio, Y. Xiong, Y. Yin and Y. Xia, Adv. Mater., 2006, 18, 1745-1749.

18 J. Yang, L. Lu, H. Wang, W. Shi and H. Zhang, Cryst. Growth Des., 2006, 6, 2155-2158.

19 B. Wiley, Y. Sun and Y. Xia, Acc. Chem. Res., 2007, 40, 10671076.

20 Y. Xiong, A. R. Siekkinen, J. Wang, Y. Yin, M. J. Kim and Y. Xia, J. Mater. Chem., 2007, 17, 2600-2602.

21 X. Zou, E. Ying, H. Chen and S. Dong, Colloids Surf., A, 2007, 303, 226-234.

22 I. Pastoriza-Santos and L. M. Liz-Marzan, J. Mater. Chem., 2008, 18, 1724-1737.

23 Y. Xia, Y. Xiong, B. Lim and S. E. Skrabalak, Angew. Chem., Int. Ed., 2009, 48, 60-103.

24 M. Liong, B. France, K. A. Bradley and J. I. Zink, Adv. Mater., 2009, 21, 1684-1689.

$25 \mathrm{P}$. Totaro and M. Rambaldini, Interactive cardiovascular thoracic surgery, 2009, 8, 153-154.

26 J. Jain, S. Arora, J. M. Rajwade, P. Omray, S. Khandelwal and K. M. Paknikar, Mol. Pharm., 2009, 6, 1388-1401.
27 F. Cheng, J. W. Betts, S. M. Kelly and A. L. Hector, Mater. Sci. Eng., $C, 2015,46,530-537$.

28 F. Cheng, J. W. Betts, S. M. Kelly, J. Schaller and T. Heinze, Green Chem., 2013, 15, 989-998.

29 D. Breitwieser, M. M. Moghaddam, S. Spirk, M. Baghbanzadeh, T. Pivec, H. Fasl, V. Ribitsch and C. O. Kappe, Carbohydr. Polym., 2013, 94, 677-686.

30 H. J. Klasen, Burns, 2000, 26, 131-138.

31 M. Rai, A. Yadav and A. Gade, Biotechnol. Adv., 2009, 27, 7683.

32 Q. L. Feng, J. Wu, G. Q. Chen, F. Z. Cui, T. N. Kim and J. O. Kim, J. Biomed. Mater. Res., 2000, 52, 662-668.

33 H. Y. Song, k. k. Ko, I. H. Oh and B. T. Lee, Eur. Cells Mater., 2006, 11, 58.

34 J. R. Morones, J. L. Elechiguerra, A. Camacho, K. Holt, J. B. Kouri, J. T. Ramirez and M. J. Yacaman, Nanotechnology, 2005, 16, 2346-2353.

35 F. Raimondi, G. G. Scherer, R. Kotz and A. Wokaun, Angew. Chem., Int. Ed. Engl., 2005, 44, 2190-2209.

36 P. Gong, H. M. Li, X. X. He, K. M. Wang, J. B. Hu, W. H. Tan, S. C. Zhang and X. H. Yang, Nanotechnology, 2017, 18, 285604.

37 S. Gupta, P. Uhlmann, M. Agrawal, S. Chapuis, U. Oertel and M. Stamm, Macromolecules, 2008, 41, 2874-2879.

38 A. Gual, C. Godard, S. Castillon, D. Curulla-Ferre and C. Claver, Catal. Today, 2012, 183, 154-171.

39 C. S. S. R. Kumar, Magnetic Nanomaterials, Wiley-VCH, Weinheim, 2009.

40 M. A. Neouze and U. Schubert, Monatsh. Chem., 2008, 139, 183-195.

41 N. S. Alahmadi, J. W. Betts, F. Cheng, M. G. Francesconi, S. M. Kelly, A. Kornherr, T. J. Prior and J. D. Wadhawan, RSC Adv., 2017, 7, 20020-20026.

42 C. Amature, Chem.-Eur. J., 2008, 14, 8615-8623.

43 M. N. Nadagouda and R. S. Varma, Biomacromolecules, 2007, 8, 2762-2767.

44 J. Cai, S. Kimura, M. Wada and S. Kuga, Biomacromolecules, 2009, 10, 87-94.

45 R. Jung, Y. Kim, H. S. Kim and H. J. Jin, J. Biomater. Sci., Polym. Ed., 2009, 20, 311-318.

46 Y. Shin, I.-T. Bae, B. W. Arey and G. J. Exarhos, J. Phys. Chem. C, 2008, 112, 4844-4848.

47 T. Maneerung, S. Tokura and R. Rujiravanit, Carbohydr. Polym., 2008, 72, 43-51.

48 S. M. Li, N. Jia, J. F. Zhu, M. G. Ma, F. Xu, B. Wang and R. C. Sun, Carbohydr. Polym., 2011, 83, 422-429.

49 F. Cheng, J. W. Betts, T. Heinze, S. M. Kelly and A. Koschella, Green Chem., 2013, 15, 989-998.

50 F. Moosdeen, J. D. Williams and A. Secker, J. Antimicrob. Chemother., 1988, 21, 439-443.

51 M. Pollini, M. Russo, A. Licciulli, A. Sannino and A. Maffezzoli, J. Mater. Sci.: Mater. Med., 2009, 20, 2361-2366. 52 T. Theivasanthi and M. Alagar, Nano Biomed. Eng., 2012, 4(2), 58-65. 